\title{
Status of SARS-CoV-2 in cerebrospinal fluid of patients with COVID-19 and stroke
}

\author{
Fadi Al Saiegh 이, ${ }^{1}$ Ritam Ghosh, ${ }^{1}$ Adam Leibold, ${ }^{1}$ Michael B Avery, ${ }_{1}^{1}$ \\ Richard F Schmidt, ${ }^{1}$ Thana Theofanis, ${ }^{1}$ Nikolaos Mouchtouris, ${ }^{1}$ Lucas Philipp, ${ }^{1}$ \\ Stephen C Peiper, ${ }^{2}$ Zi-Xuan Wang, ${ }^{3}$ Fred Rincon, ${ }^{1}$ Stavropoula I Tjoumakaris, ${ }^{1}$ \\ Pascal Jabbour, ${ }^{1}$ Robert H Rosenwasser, ${ }^{1}$ M. Reid Gooch ${ }^{1}$
}

\begin{abstract}
${ }^{1}$ Department of Neurological Surgery, Thomas Jefferson University, Philadelphia, Pennsylvania, USA 2Pathology, Anatomy, and Cell Biology, Thomas Jefferson University, Philadelphia, Pennsylvania, United States ${ }^{3}$ Surgery \& Pathology, Molecular \& Genomic Pathology Laboratory, Thomas Jefferson University, Philadelphia, Pennsylvania, United States
\end{abstract}

\section{Correspondence to} Dr M. Reid Gooch, Department of Neurological Surgery, Thomas Jefferson University, Philadelphia PA 19107, Pennsylvania, USA; reid.gooch@ jefferson.edu

Received 15 April 2020 Accepted 17 April 2020 Published Online First 30 April 2020

Check for updates

(C) Author(s) (or their employer(s)) 2020. No commercial re-use. See rights and permissions. Published by BMJ.

To cite: Al Saiegh F, Ghosh R, Leibold A, et al. J Neurol Neurosurg Psychiatry

2020:91:846-848.

\section{ABSTRACT}

Background Emergence of the novel corona virus (severe acute respiratory syndrome (SARS)-CoV-2) in December 2019 has led to the COVID-19 pandemic. The extent of COVID-19 involvement in the central nervous system is not well established, and the presence or the absence of SARS-CoV-2 particles in the cerebrospinal fluid (CSF) is a topic of debate.

Case description We present two patients with COVID-19 and concurrent neurological symptoms. Our first patient is a 31-year-old man who had flu-like symptoms due to COVID-19 and later developed an acute-onset severe headache and loss of consciousness and was diagnosed with a Hunt and Hess grade 3 subarachnoid haemorrhage from a ruptured aneurysm. Our second patient is a 62-year-old woman who had an ischaemic stroke with massive haemorrhagic conversion requiring a decompressive hemicraniectomy. Both patients' CSF was repeatedly negative on real-time PCR analysis despite concurrent neurological disease.

Conclusion Our report shows that patients' CSF may be devoid of viral particles even when they test positive for COVID-19 on a nasal swab. Whether SARS-CoV-2 is present in CSF may depend on the systemic disease severity and the degree of the virus' nervous tissue tropism and should be examined in future studies.

\section{INTRODUCTION}

The emergence of the novel corona virus (severe acute respiratory syndrome (SARS)-CoV-2) in December 2019 in Wuhan, China, has led to a global pandemic with almost unprecedented global health and economic consequences. In infected patients, the virus primarily causes severe respiratory symptoms that are collectively coined as COVID-19. These symptoms can culminate in a cytokine storm resulting in acute respiratory distress syndrome (ARDS). ${ }^{1}$ However, multiple reports have raised concerns about the virus' tendency to invade the central nervous system (CNS). ${ }^{2}$ The spectrum of CNS involvement ranges from meningitis or encephalitis to ischaemic stroke believed to be caused by thrombophilia. ${ }^{4}$ The presence or the absence of SARS-CoV-2 in cerebrospinal fluid (CSF) is not established when a patient with a positive nasal swab has concurrent neurological symptoms.
In this report, we present two patients with COVID-19 and concurrent neurological symptoms who repeatedly tested negative for SARS-CoV-2 RNA in their CSF. Our first patient had a Hunt and Hess $(\mathrm{H} \& \mathrm{H})$ grade 3 aneurysmal subarachnoid haemorrhage (SAH), and our second patient had an ischaemic stroke with massive haemorrhagic conversion.

\section{CASE DESCRIPTION}

\section{Patient 1}

The patient is a 31-year-old man with no recent travel and no medical history who initially started having malaise, mild fever, cough and arthralgia for about 1 week. He remained at home but was taken to an outside institution after sudden onset of headache and then loss of consciousness. A head CT scan was obtained and showed SAH centred in the posterior fossa, including the fourth ventricle (figure 1A). He regained consciousness at the outside hospital and was maintaining his airway with good levels of oxygenation. He was then referred to our institution for management of the SAH. On arrival, he was noted to be lethargic and his head CT showed hydrocephalus (figure 1B). Per our institutional protocol, we obtained informed patient consent from his wife and proceeded to intubate the patient for placement of an external ventricular drain (EVD) to drain the CSF and to relieve the hydrocephalus and treat the aneurysm. Due to concurrent flu-like symptoms, a nasal swab was sent to test for COVID-19. The patient had a cerebral angiogram that showed a right-sided ruptured dissecting posterior-inferior cerebellar artery aneurysm that was treated with a flow-diverting stent (figure 1C). The procedure was uneventful and the patient was extubated on postoperative day (POD) 1 .

On POD 2, the results returned positive for COVID-19. The patient had no focal neurological deficits but showed signs of confusion. Therefore, due to concern for potential SARS-CoV2-associated encephalitis, CSF was sent for testing from the patient's EVD. A qualitative real-time PCR for SARS-CoV-2 returned negative twice. The patient's confusion gradually improved, and he was discharged to rehabilitation. Of note, the patient was tested via nasal swab for COVID-19 multiple times throughout his stay and was persistently positive. 

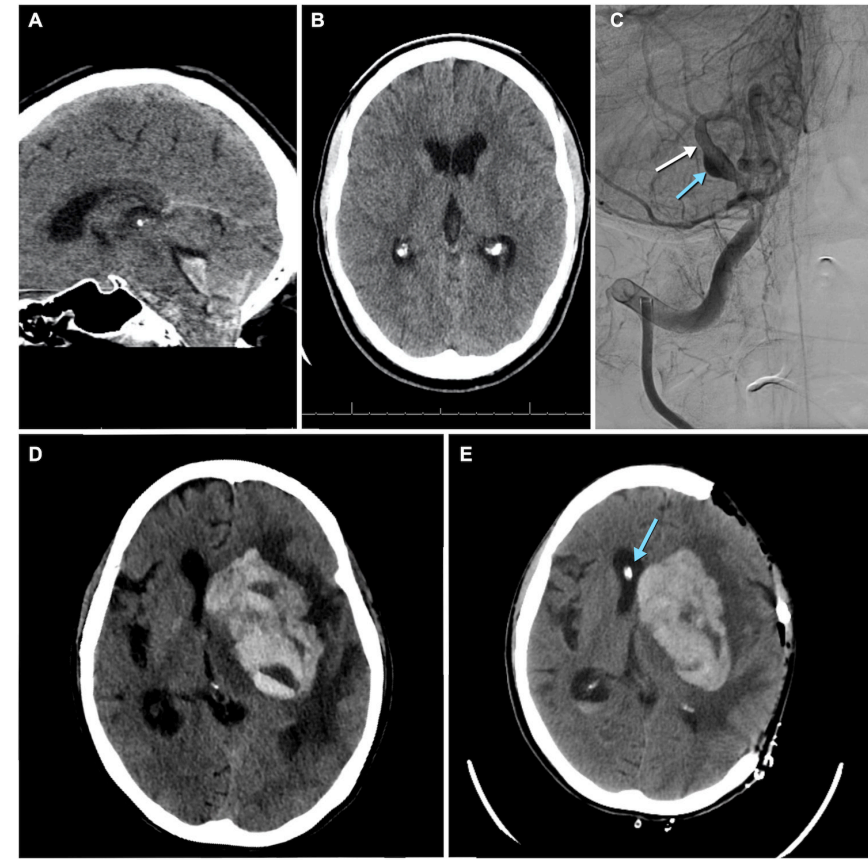

Figure 1 Patient 1: top panel. Patient 2: bottom panel. Sagittal head CT shows blood in the posterior fossa, particularly in the fourth ventricle. Axial head CT shows hydrocephalus. Lateral right-sided vertebral artery angiogram after deployment of the flow-diverting stent (white arrow) and expected stasis in the aneurysm (blue arrow). Axial head CT with a large left intracerebral haemorrhage with surrounding oedema. Postoperative axial head CT showing removal of the bone flap and placement of the right external ventricular drain (arrow).

\section{Patient 2}

Our second patient is a 62-year-old woman who had an acuteonset right hemiparesis and aphasia, and her CT angiogram revealed a left middle cerebral artery occlusion, for which she underwent mechanical thrombectomy with full recanalisation. She was discharged to rehabilitation in late March 2020, but 10 days after discharge, she returned with a change in mental status, and her head CT demonstrated haemorrhagic conversion with midline shift and obstructive hydrocephalus (figure 1D) requiring a decompressive hemicraniectomy and EVD placement (figure 1E). Postoperatively, the patient remained intubated due to poor mental status. As part of evaluation for tracheostomy, the patient underwent COVID-19 testing via a nasal swab, which was positive in the absence of overt symptoms, such as persistent fever or an ARDS-like picture on chest X-ray. Since the patient continued to have an EVD in place, CSF was sent for SARS-CoV-2 testing to rule out CNS involvement, and realtime PCR was negative on two consecutive samples. Of note, the patient was retested via nasal swab 4 days after her first positive result and her nasal swab was negative for COVID-19, indicating recovery. Individual patient consent was obtained for the publication of this report.

\section{DISCUSSION}

Due to the worldwide spread of the novel strain of corona virus (SARS-CoV-2) causing respiratory disease dubbed COVID-19, there is newfound attention on its widespread effects on the medical community. Neurosurgery is no exception, as clinical practices had to be adapted to effectively treat patients in the pandemic while also taking precautions for the safety of the care teams involved. Here, we present two cases of patients with intracranial haemorrhage-including the first reported case of aneurysmal SAH-both of whom tested positive for COVID-19.

It has been reported in the literature that SARS-CoV may invade the CNS, and due to the high similarity between SARS-COV and SARS-CoV-2, it is possible that the latter possesses the same potential..$^{5}$ The initial report of the COVID-19 outbreak by Zhou et $a l^{6}$ corroborated previous reports ${ }^{7}$ and confirmed that SARS-CoV-2 uses the same receptor for cell entry as SARS-CoV, namely, membrane-bound ACE II (ACE2). ACE2 has been demonstrated to be expressed in neurons, as well as endothelial and arterial smooth muscle cells in the brain, ${ }^{8}$ potentially allowing SARS-CoV-2 to cross the blood-brain barrier and affect the CNS. This may allow the virus to cause viral meningitis or, in the case of our patients, could lead to intracranial or aneurysmal haemorrhage. Additionally, autopsy reports have revealed brain tissue oedema and neuronal degeneration in deceased patients, and experimental animal studies using SARS-CoV or Middle East respiratory syndrome- $\mathrm{CoV}$ have shown that virus particles can be detected in specific brain areas, such as the thalamus and the brainstem. ${ }^{10}{ }^{11}$ In a case study with 214 patients with confirmed COVID-19 infections, 36.4\% showed neurological symptoms such as dizziness, headache and confusion, with $5.7 \%$ of these patients having cerebrovascular disease, including ischaemic stroke and intracerebral haemorrhage. ${ }^{12}$

Perhaps the most striking evidence suggesting nervous tissue tropism comes as a by-product of two case reports from Japan and China, in which patients with COVID-19 showed signs of meningitis. In both patients, PCR analysis of the CSF samples turned positive for COVID-19, showing that the virus has the potential to cross the blood-brain barrier. ${ }^{23}$

In our first patient, who had no risk factors for cerebral aneurysms, his sentinel event was preceded by days of fatigue, dry cough and general malaise. He then had sudden-onset loss of consciousness and was diagnosed at a nearby hospital with $\mathrm{H} \& \mathrm{H}$ grade $3 \mathrm{SAH}$ and presented to our institution with lethargy and hydrocephalus, and later tested positive for COVID-19.

Our second patient was found to have COVID-19 as part of the preoperative work-up for tracheostomy placement. This illustrates the challenge of treating neurosurgical patients with this virus, as many patients are unable to describe symptoms prior to presentations.

In published reports on SARS-CoV-2, a cytokine storm has been postulated, ${ }^{1}{ }^{13}$ which could result in cerebrovascular disease. Furthermore, severe cases of COVID-19 have shown elevated D-dimer levels and thrombocytopaenia, rendering patients prone to cerebrovascular events, both thrombotic and haemorrhagic. ${ }^{14-16}$ Whether or not our first patient's aneurysm rupture was directly related to his infection is difficult to ascertain. However, it is possible that in a patient without any significant risk factors, the cytokine storm, coupled with the sympathetic drive in an attempt to combat the infection, may have had a role in the aneurysm formation and rupture.

Furthermore, since our second patient was an asymptomatic carrier, it is unclear when her first exposure occurred. However, due to the proximity of her neurological events, it is likely that she carried the virus during the initial stroke event and her subsequent haemorrhage. Therefore, it is conceivable that the thrombotic event-in the absence of other risk factors-is secondary to derangements in the coagulation cascade related to COVID19 , and the following haemorrhagic event may be related to the aforementioned cytokine storm.

While it is nearly impossible to link COVID-19 to intracranial pathology, CSF analysis can be used as an objective marker for CNS involvement. In both of our patients who had 
ventriculostomies placed, CSF samples were sent to evaluate for viral CNS involvement. Specific SARS-CoV-2 RNA was not detected in both patients' CSF on two separate real-time PCR runs. This is in direct contradiction to a case report by Moriguchi et al, which showed no SARS-CoV-2 in the nasal swab but only in the CSF. ${ }^{2}$

Both of our patients had relatively mild respiratory symptoms without progression to ARDS. Our first patient was able to be extubated on POD 1 and did not require any extensive ventilator support. Our second patient was able to be weaned from ventilator support and never required ARDS treatment. We suspect that, in light of a non-fulminant course of the disease, there was no disruption of the blood-brain barrier that would allow for SARS-CoV-2 to cross into the CSF space.

\section{CONCLUSION}

We report the absence of SARS-CoV-2 in the CSF of two patients with concurrent severe neurological symptoms but a non-fulminant respiratory disease course. In such cases, an underlying inflammatory and hypercoagulable state may incite cerebrovascular disease without disruption of the blood-brain barrier. We encourage further CSF analysis in patients with confirmed COVID-19 as this will give us a better understanding of the virus's tissue tropism.

Contributors FAS and RG: conceptualisation and methodology; SP, ZW, NM, RG, AL, MBA, RFS, TT, NM, LP, FR, SIT, PJ and RHR: data curation and formal analysis; RG: study supervision; FAS and LP: visualisation; FAS: original draft preparation;All authors: writing, review and editing.

Funding The authors have not declared a specific grant for this research from any funding agency in the public, commercial or not-for-profit sectors.

Competing interests None declared.

Patient consent for publication Obtained.

Provenance and peer review Not commissioned; internally peer reviewed.

ORCID iD

Fadi Al Saiegh http://orcid.org/0000-0002-1544-4910

\section{REFERENCES}

1 Chen C, Zhang XR, Ju ZY, et al. [Advances in the research of cytokine storm mechanism induced by Corona Virus Disease 2019 and the corresponding immunotherapies]. Zhonghua Shao Shang Za Zhi 2020;36:E005.

2 Moriguchi T, Harii N, Goto J, et al. A first case of meningitis/encephalitis associated with SARS-Coronavirus-2. Int J Infect Dis 2020;94:55-8.

3 Li Y-C, Bai W-Z, Hashikawa T. The neuroinvasive potential of SARS-CoV2 may play a role in the respiratory failure of COVID-19 patients. J Med Virol 2020. doi:10.1002/ jmv.25728. [Epub ahead of print: 27 Feb 2020] (published Online First: 2020/02/28).

4 Poyiadji N, Shahin G, Noujaim D, et al. COVID-19-associated acute hemorrhagic necrotizing encephalopathy: CT and MRI features. Radiology 2020:201187 (published Online First: 2020/04/02).

5 Wu Y, Xu X, Chen Z, et al. Nervous system involvement after infection with COVID-19 and other coronaviruses. Brain Behav Immun 2020. doi:10.1016/j.bbi.2020.03.031. [Epub ahead of print: 30 Mar 2020] (published Online First: 2020/04/03).

6 Zhou P, Yang X-L, Wang X-G, et al. A pneumonia outbreak associated with a new coronavirus of probable bat origin. Nature 2020;579:270-3.

7 Ge X-Y, Li J-L, Yang X-L, et al. Isolation and characterization of a bat SARS-like coronavirus that uses the ACE2 receptor. Nature 2013;503:535-8.

8 Xia H, Lazartigues E. Angiotensin-Converting enzyme 2 in the brain: properties and future directions. J Neurochem 2008;107:1482-94.

9 Ding $\mathrm{Y}$, Wang $\mathrm{H}$, Shen $\mathrm{H}$, et al. The clinical pathology of severe acute respiratory syndrome (SARS): a report from China. J Pathol 2003;200:282-9.

10 Hu D, Zhu C, Ai L, et al. Genomic characterization and infectivity of a novel SARS-like coronavirus in Chinese bats. Emerg Microbes Infect 2018;7:1-10.

11 Li K, Wohlford-Lenane C, Perlman S, et al. Middle East respiratory syndrome coronavirus causes multiple organ damage and lethal disease in mice transgenic for human dipeptidyl peptidase 4. J Infect Dis 2016;213:712-22.

12 Mao L, Wang M, Chen S, et al. Neurological manifestations of hospitalized patients with COVID-19 in Wuhan, China: a retrospective case series study. SSRN Journal 2020.

13 Mehta P, McAuley DF, Brown M, et al. COVID-19: consider cytokine storm syndromes and immunosuppression. Lancet 2020;395:1033-4.

14 Driggin E, Madhavan MV, Bikdeli B, et al. Cardiovascular considerations for patients, health care workers, and health systems during the coronavirus disease 2019 (COVID-19) pandemic. J Am Coll Cardiol 2020. doi:10.1016/j. jacc.2020.03.031. [Epub ahead of print: 18 Mar 2020] (published Online First: 2020/03/24).

15 Tang N, Bai H, Chen $\mathrm{X}$, et al. Anticoagulant treatment is associated with decreased mortality in severe coronavirus disease 2019 patients with coagulopathy. J Thromb Haemost 2020

16 Zhang Y, Xiao M, Zhang S, et al. Coagulopathy and antiphospholipid antibodies in patients with Covid-19. N Engl J Med 2020. doi:10.1056/NEJMc2007575. [Epub ahead of print: 08 Apr 2020] (published Online First: 2020/04/09). 